\title{
Airport Noise Regulation, Airline Service Quality, and Social Welfare
}

\author{
by \\ Jan K. Brueckner \\ Department of Economics \\ University of California, Irvine \\ 3151 Social Science Plaza \\ Irvine, CA 92697 \\ e-mail: jkbrueck@uci.edu \\ and \\ Raquel Girvin \\ Program in Transportation Science \\ University of California, Irvine \\ Irvine, CA 92697 \\ e-mail: rgirvin@uci.edu
}

September 2006

\begin{abstract}
This paper explores the impact of airport noise regulation on airline service quality and airfares. It also characterizes the socially optimal stringency of noise limits, taking both noise damage and the various costs borne by airlines and their passengers into account. The analysis also investigates the effect of noise taxes, as well as the optimal level level of such taxes. Along with the companion paper by Girvin (2006a), this work represents the first complete theoretical investigation into the economics of airport noise regulation using a model where the interests of the key relevant stakeholders are captured.
\end{abstract}




\title{
Airport Noise Regulation, Airline Service Quality, and Social Welfare
}

by

\author{
Jan K. Brueckner and Raquel Girvin
}

\section{Introduction}

Despite dramatic reductions over the years in the noise produced by individual aircraft, airport noise remains a critical public policy issue today. Moreover, given the expected increases in airline traffic and airport operations over the next decades, the noise issue will continue to be a source of dissension. The nature of the problem is evident in the ongoing controversy surrounding the planned expansion and reorientation of Chicago's O'Hare airport. While the expansion requires demolition of several hundred properties, it also reorients the airport's flight paths, so that a new set of households will be exposed to noise (see McMillen (2004)). Both of these anticipated effects have led to vociferous opposition to the expansion from nearby residents, who have attempted to block the plan in court, despite its recent approval by the Federal Aviation Administration (FAA). Similarly, in Orange County, California, concerns about noise exposure blocked the construction of a new international airport on a decommissioned military airbase, even with a shortage of airport capacity in the region (Kranser (2002)). At nearby John Wayne Airport, departing flights must practice a steep, high-power climb maneuver to quickly gain altitude before passing over the high-income community of Newport Beach, and noise concerns in that community continue to limit daily flight volume at the airport. ${ }^{1}$

The dramatic gains in aircraft "quietness" over the jet age, which have ironically accompanied ongoing noise concerns, are illustrated by comparing noise from a recent-vintage Boeing 737-700 and a 1967-vintage 727-200. The newer aircraft produces only one-third as much perceived takeoff noise as its predecessor, despite similar passenger capacities. Because of such gains in quietness, the number of U.S. residents exposed to significant aircraft noise fell by a factor of 16 between 1975 and 2000 despite a more than three-fold increase in airline traffic over the period. However, even with such gains in noise abatement, Figure 1 shows sharply 
growing trends in various airport noise limits, such as operational curfews, noise quotas, and noise surcharges, in the U.S. Such measures are even more widespread in Europe, as discussed by Girvin (2000c).

Noise restrictions are likely to have important impacts on airline service quality and airfares. Service quality may fall as various operational limits restrict flight frequency, and the expense of making aircraft quieter, which raises their purchase price and operating cost, may be passed on in higher airfares. Despite these possible linkages, the airline economics literature contains no comprehensive analysis of the effect of noise regulation on airline service quality and fares. ${ }^{2}$ Because of this absence, no proper analysis of optimal noise regulations, which maximize social welfare taking into account impacts on airlines and their passengers as well as noise victims, has been possible. The purpose of this paper is to provide the missing analysis through the use of a highly stylized, but suggestive, theoretical model.

The analysis draws on the scheduling model of Brueckner (2004), where higher flight frequency benefits passengers by reducing "schedule delay" (allowing departures at moreconvenient times) while generating higher total noise. Noise per aircraft, denoted $n$, can be reduced at a cost, which rises with aircraft size given that quieting a larger plane is more expensive. The airline is viewed as choosing both $n$ and aircraft size, along with flight frequency and fares, to maximize profit subject to noise regulations. The manufacturer responds to the resulting demand for aircraft quietness in its design decisions.

The analysis considers two different regulatory regimes involving explicit noise constraints, along with an alternative regime where airlines pay noise taxes. The first type of noise constraint imposes a direct limit on noise per aircraft, with the constraint written as $n \leq \bar{n}$, where $\bar{n}$ is the limit. Note that, under this constraint, $n$ is removed as a choice variable for the airline. The $\bar{n}$ limit is analogous to the FAA noise certification standard, which governs quietness levels for new aircraft while also requiring retrofitting of older, noisier planes. ${ }^{3}$

The second type of constraint is a cumulative noise limit at an airport, which is written $n f \leq L$, where $f$ is flight frequency (the number of flights) and $L$ is total allowed noise for each airline. Note that an airline has flexibility in meeting a cumulative limit because total noise depends on both $n$ and flight frequency. This type of constraint, among other noise 
restrictions, is imposed by Amsterdam's Schiphol Airport (AMS), London's Heathrow Airport (LHR), and Long Beach Airport (LGB) in California. ${ }^{4}$ Even though cumulative noise limits are not widespread currently, Figure 1 suggests that they are likely to become more common as time passes, making an understanding of their effects important.

Under noise taxation, explicit constraints are removed, but the airline instead pays a tax of $t$ per unit of noise, so that its total tax liability is tnf. Although noise taxes are not used much in the U.S., they are more common elsewhere, being levied through landing-fee adjustments that depend on an aircraft's noise level. For institutional background, see Nero and Black (2000) and Morell and Lu (2000).

In the analysis, the effect of each of the three regulatory regimes is considered in isolation, ignoring the fact that different regimes often coexist in reality. This potentially unrealistic approach is meant to gain insight into the economics of the regimes, and its practical lessons are considered in the conclusion. Another unrealistic element is the linear fashion in which noise is added across flights under the cumulative limit. Under the actual regulations, noise is added in a semi-logarithmic fashion, which makes total noise more sensitive to noise per flight than to the number of flights. ${ }^{5}$ While incorporating this feature would complicate the analysis, the main qualitative conclusions are likely to be unaffected.

The analysis solves the airline's profit-maximization problem under cumulative and peraircraft noise regulation and under the noise-tax regime. Comparative-static analysis shows the effects of parameter changes on the airline's choice variables, results that are immediate given that the model generates closed form solutions for all the variables. In addition, a key equivance result between noise taxation and cumulative noise regulation is established.

While the cumulative or per-aircraft noise limits are treated as parameters in the comparative-static analysis, the next step is to consider the social planner's problem, where $L$ or $\bar{n}$ is chosen optimally, taking into account the environmental damage from noise. Even though the choice of a regulatory regime (cumulative vs. per-aircraft noise regulation) is immaterial in characterizing the first-best social optimum, the two regimes generate different outcomes in the second-best case, where the planner must accept the (inefficient) profit-maximizing choices of the airline. An interesting question is then which regime yields a higher welfare level. This 
question cannot be addressed analytically, but it is investigated via numerical analysis. The analysis also characterizes the noise tax rates that support the first-best and second-best social optima.

The entire analytical agenda described above can be carried out either under a monopoly market structure, with a single airline, or in an oligopoly setting. Since the monopoly case has already been analyzed by Girvin (2006a), the present paper analyzes an oligopoly model, with the specific case of a duopoly considered. As seen below, the duopoly setup is actually a special case of the monopoly model, and the results for this latter model are briefly summarized. The duopoly model is drawn from the analysis of Brueckner and Flores-Fillol (2006), and its key element is idiosyncratic airline brand loyalty on the part of consumers, which governs competition between the two duopoly carriers.

The plan of the paper is as follows. Section 2 develops the basic model. Section 3 introduces the cumulative and per-aircraft noise constraints and solves the airline profit-maximization problem under these two different regimes. Section 4 analyzes noise taxation, section 5 carries out the welfare analysis, and section 6 offers conclusions.

\section{The Model}

To start the analysis, it is useful to focus on airline costs. Following Brueckner (2004), the cost of each flight, exclusive of noise-abatement cost, is given by $\theta+\tau s$, where $s$ equals seats per flight. Each flight thus entails a fixed $\operatorname{cost} \theta$ as well as a variable $\operatorname{cost} \tau$ per seat. While this specification may not be completely realistic, it captures the fact that an airline's cost per seat (given by $\theta / s+\tau$ ) falls as aircraft size increases. Multiplying by flight frequency yields a total cost expression, $f(\theta+\tau s)$.

Noise-abatement cost per flight, which is embodied in the aircraft design, is assumed to depend on both aircraft size and quietness, being written $g(s, n)$. The function $g$ is increasing in $s$, indicating that it costs more to achieve a given level of quietness for a larger aircraft. In addition, $g$ is decreasing in $n$, noise per aircraft, indicating that a quieter aircraft of a given size generates higher costs, partly through lower fuel efficiency. Since noise abatement affects both the purchase price and operating cost of an aircraft, $g$ should be viewed as giving the 
annualized abatement cost.

The function $g$ is assumed to be homogeneous of degree zero, so that proportional increases in aircraft size and noisiness leave abatement costs unchanged. A particular functional form is then imposed for analytical tractability, with $g(s, n) \equiv \epsilon s / n$, where $\epsilon>0$ is the abatementcost parameter. This form embodies the above assumptions on $g$ 's partial derivatives, and its simplicity yields closed-form solutions for the variables of interest. With total noise abatement cost equal to $f \epsilon s / n$, the airline's full cost is written

$$
f(\theta+\tau s)+\epsilon f s / n=\theta f+(\tau+\epsilon / n) f s
$$

Turning to the demand side of the model, the analysis focuses on a single city-pair market served by two airlines, although the extension to multiple markets is straightforward. Utility for a consumer traveling by air between the cities is given by $c+$ travel benefit-schedule delay cost, where $c$ is consumption expenditure and schedule delay measures the difference between the actual and desired departure times. To represent schedule delay and its cost, suppose consumers must commit to travel before knowing their preferred departures times, which are uniformly distributed around a circle. Letting $T$ denote the time circumference of the circle, consumer utility from flying on airline 1 then depends on expected schedule delay, which equals $T / 4 f_{1}$, where $f_{1}$ is number of (evenly spaced) flights operated by the airline $\left(T / 4 f_{2}\right.$ is the analogous expression for airline 2). These expressions give the expected interval between the desired departure time and the nearest flight time. Schedule delay cost is then given by a disutility parameter $\delta>0$ times the above expressions, thus equaling $\delta T / 4 f_{i} \equiv \gamma / f_{i}$ for airline $i, i=1,2$, where $\gamma=\delta T / 4$.

Travel benefit has two components: $b$, equal to the gain from air travel, and $a$, an airline brand-loyalty variable, which gives the additional gain from using airline 1 (relative to travel on airline 2). Brand loyalty varies across consumers, with a uniformly distributed over the interval $[-\alpha / 2, \alpha / 2]$, where $\alpha>0$, so that half the consumers prefer airline 1 and half prefer airline 2 .

For consumers using airline $i$, consumption expenditure equals $y-p_{i}$, where $y$ is income, assumed to be uniform across consumers without loss of generality, and $p_{i}$ is airline $i$ 's fare. 
Utility from travel on airline 1 is thus given by $y-p_{1}+b+a-\gamma / f_{1}$, and utility from travel on airline 2 is given by the analogous expression with $a=0$. Assuming that the consumer undertakes air travel, his choice is between the two airlines, and airline 1 will be chosen when

$$
y-p_{1}+b+a-\gamma / f_{1}>y-p_{2}+b-\gamma / f_{2},
$$

or when

$$
a>p_{1}-p_{2}+\gamma / f_{1}-\gamma / f_{2} .
$$

Thus, for the consumer to choose airline 1, brand loyalty to 1 must be at least as large as the fare plus delay-cost difference between airlines 1 and 2. Otherwise, conditional on flying, the consumer will choose airline 2 .

To compute airline 1's traffic using (3), let the consumer population size be normalized to unity. Then, recalling that $a$ is uniformly distributed over the range $[-\alpha / 2, \alpha / 2]$, airline 1 's traffic is given by

$$
q_{1}=\int_{p_{1}-p_{2}+\gamma / f_{1}-\gamma / f_{2}}^{\alpha / 2} \frac{1}{\alpha} d a
$$

where $1 / \alpha$ gives the density of $a$. Carrying out the integration in (4) yields

$$
q_{1}=\frac{1}{2}-\frac{1}{\alpha}\left[p_{1}-p_{2}+\gamma / f_{1}-\gamma / f_{2}\right]
$$

with $q_{2}$ given by the analogous expression with the 1 and 2 subscripts interchanged. Note from (5) that airline 1 loses traffic when its fare rises or its frequency falls, while it gains traffic when $p_{2}$ rises or $f_{2}$ falls. ${ }^{6}$

The monopoly model, analyzed by Girvin (2006a), is generated by considering an "outside option" not involving air travel, which could represent use of a different transport mode or not traveling at all. It yields benefits $e$ net of any fare paid or schedule delay incurred and thus a utility level of $y+e$. The monopoly model emerges when a consumer who is indifferent between the airlines, with $a=0$, prefers the outside option to air travel. Normalizing $e$ to zero, this 
outcome requires $y-p_{1}+b-\gamma / f_{1}<y$, or $b<p_{1}+\gamma / f_{1}$, and $b<p_{2}+\gamma / f_{2}$. While consumers with $a$ values near zero will not fly when these equalities hold, stronger airline brand loyalty can induce air travel. For example, a passenger loyal to airline 1 will find air travel and the outside option equally attractive when his loyalty value satisfies $y-p_{1}+b+a-\gamma / f_{1}=y$, or $a=p_{1}+\gamma / f_{1}-b .^{7}$ Thus, consumers with $a$ values larger than

$$
\widehat{a}=p_{1}+\gamma / f_{1}-b
$$

will then strictly prefer travel on airline 1 to the outside option, so that airline 1's traffic is equal to 8

$$
q_{1}=\int_{\widehat{a}}^{\alpha / 2} \frac{1}{\alpha} d a=\frac{1}{2}-\frac{p_{1}+\gamma / f_{1}-b}{\alpha} .
$$

Since airline 2's fare and frequency choices do not appear in (7), competition between the carriers is not present, with airline 1 effectively becoming a monopolist for passengers with positive $a$ values (airline 2 becomes a monopolist for negative- $a$ passengers).

For the duopoly case to be relevant, $b \geq p+\gamma / f$ must hold at the $p$ and $f$ values that emerge in a symmetric duopoly equilibrium. Otherwise, passengers with $a$ values near zero will prefer the outside option, making the monopoly case relevant instead. Accordingly, $b$ is assumed to be large enough to ensure satisfaction of the above inequality, with the exact lower bound discussed below. ${ }^{9}$ Results for the monopoly case, which are presented by Girvin (2006a), are summarized once the duopoly analysis is complete. ${ }^{10}$

It should be noted that, while a monopoly airline's equilibrium traffic level depends on its fare and frequency, as can be seen from (7), each duopoly airline carries an equilibrium traffic level of $1 / 2$ in a symmetric equilibrium regardless of its choices. From $(5), q_{1}=1 / 2$ holds when fares and frequencies are the same across carriers. In reaching the equilibrium, however, each carrier takes into account traffic gains and losses to the other carrier as it adjusts its choice variables, as seen below. But since no consumer is assumed to choose the outside option, the carriers battle for a fixed amount of total traffic, which is split evenly between them in equilibrium. This feature of the model accounts the simplicity of the ensuing analysis and the 
emergence of clear-cut comparative-static effects, which are mostly absent in the monopoly case.

With the traffic solution in (5) relevant for the duopoly case, it can be combined with the previous cost expression in (1) to yield profit. Multiplying (5) by $p_{1}$ and subtracting (1) (with subscripts added), airline 1's profit is equal to

$$
\begin{gathered}
\pi_{1}=p_{1} q_{1}-\theta f_{1}-\left(\tau+\epsilon / n_{1}\right) f_{1} s_{1} \\
=p_{1} q_{1}-\theta f_{1}-\left(\tau+\epsilon / n_{1}\right) q_{1} \\
=\left(p_{1}-\tau-\epsilon / n_{1}\right)\left(\frac{1}{2}-\frac{1}{\alpha}\left[p_{1}-p_{2}+\gamma / f_{1}-\gamma / f_{2}\right]\right)-\theta f_{1},
\end{gathered}
$$

Note that (8) uses the equality $f_{1} s_{1}=q_{1}$, which says that the number of flights times seats per flight equals total traffic. Using (8), the subsequent analysis considers the profit-maximization problem under the two different regulatory regimes.

\section{Airline Choices Under Noise Constraints}

\subsection{The case of a cumulative constraint}

Suppose that noise regulation takes the form of a cumulative noise constraint, which imposes a common limit on the total noise generated by each airline, being written $n_{i} f_{i} \leq L$, $i=1,2$. Since this constraint will bind in equilibrium, it can be used to eliminate $n_{1}$, which can be written $n_{1}=L / f_{1}$. Substituting in (8), the multiplicative $p_{1}-\tau-\epsilon / n_{1}$ term becomes $p_{1}-\tau-\epsilon f_{1} / L$. The resulting objective function then depends only on the choice variables $p_{1}$ and $f_{1}$.

In modeling airline decisions, an important question concerns the timing of choices. While both flight frequencies and fares can be adjusted relatively easily, fares appear to be more flexible than frequencies. As a result, under the most realistic approach, fares would be chosen conditional on frequencies, which themselves would be set in a first stage, taking the secondstage impact on fares into account (the outcome is a subgame perfect Nash equilibrium). The analysis eventually adopts this approach, but it is useful to start by considering the less- 
realistic case where fares and frequencies are chosen simultaneously in Nash fashion. Under this approach, airline 1 simultaneously sets the levels of $p_{1}$ and $f_{1}$, taking $p_{2}$ and $f_{2}$ as parametric.

The first-order conditions for the simultaneous-choice problem are

$$
\begin{gathered}
\frac{\partial \pi_{1}}{\partial p_{1}}=q_{1}-\frac{p_{1}-\tau-\epsilon f_{1} / L}{\alpha}=0 \\
\frac{\partial \pi_{1}}{\partial f_{1}}=-\frac{\epsilon}{L}\left(\frac{1}{2}-\frac{1}{\alpha}\left[p_{1}-p_{2}+\gamma / f_{1}-\gamma / f_{2}\right]\right)+\frac{\gamma\left(p_{1}-\tau-\epsilon f_{1} / L\right)}{\alpha f_{1}^{2}}-\theta=0
\end{gathered}
$$

While the derivatives $\partial^{2} \pi_{1} / \partial p_{1}^{2}$ and $\partial^{2} \pi_{1} / \partial f_{1}^{2}$ are easily seen to be negative, the remaining second-order condition (positivity of the Hessian matrix of $\pi_{1}$ ) is assumed to hold. ${ }^{11}$

Given the symmetry of the setup, the equilibrium fares and frequencies will be symmetric across carriers, with the values denoted $p$ and $f$, and each airline's traffic will equal 1/2. Imposing the latter condition in (9) and rearranging, the fare can be written

$$
p=\alpha / 2+\tau+\epsilon f / L
$$

Thus, the fare equals the full marginal cost of a seat, given by the operating cost $\tau$ plus noise abatement cost per seat $(\epsilon f / L),{ }^{12}$ plus a markup that depends on brand loyalty. Note that, as the extent of brand loyalty rises, leading to a higher $\alpha / 2$, a carrier loses less traffic from raising its fare, so that $p$ increases.

After substituting (11) into (10) and imposing symmetry, the equation can be solved for the equilibrium frequency, which equals

$$
f=\sqrt{\frac{\gamma}{2 \theta+\epsilon / L}} .
$$

This solution shows that frequency is increasing in the disutility of schedule delay $(\gamma)$ and decreasing in the fixed cost per flight $(\theta)$, both natural conclusions. Since the fare in (11) increases with frequency, $p$ is then increasing in $\gamma$ and decreasing in $\theta$. Note that the last 
conclusion, which says that $p$ is inversely related to the fixed cost per flight, is counterintuitive. The explanation is that the reduction in service quality caused by the higher $\theta$ reduces willingness to pay for air travel. Observe also that $f$ is independent of the marginal seat cost $(\tau)$ and the extent of brand loyalty $(\alpha) .{ }^{13}$

The key comparative-static question, however, concerns the effect of the noise limit $L$ on the airline's choice variables. When the noise constraint is relaxed by increasing $L,(12)$ shows that frequency rises, with the denominator expression becoming smaller. This conclusion is natural since, as $L$ rises, the airline can raise $f$ for a given $n$ without violating the constraint. With $L$ and $\epsilon$ appearing in ratio form in (12), the opposite conclusion applies to an increase in $\epsilon$ : by raising the cost per flight, a higher noise-abatement cost lowers frequency.

Turning to the impact of $L$ on $p$, the effect is not immediately apparent given that $p$ has an direct inverse dependence on $L$ from (11) as well as an indirect dependence via $f$. However, after substituting (12) into (11) and rearranging, the last term reduces to $\sqrt{\gamma \epsilon^{2} /\left(2 \theta L^{2}+\epsilon L\right)}$, which is decreasing in $L$. Therefore, relaxation of the noise constraint leads to a lower equilibrium fare, a conclusion that shows how the cost of airline travel is affected along with service quality $(f)$ by the stringency of noise regulation. Since $L$ and $\epsilon$ enter asymmetrically in the previous expression, their impacts are no longer mirror images. But it is easily seen that the expression is increasing in $\epsilon$, so that the fare naturally rises with the cost of abatement.

Aircraft quietness and size, $n$ and $s$, can be recovered from the $f$ solution, allowing further comparative-static effects to be derived. Since $s=q / f=1 / 2 f$, the parameter impacts on aircraft size are opposite to those on flight frequency. With $n=L / f$, substitution of (12) yields

$$
n=\sqrt{\frac{2 \theta L^{2}+\epsilon L}{\gamma}},
$$

so that $n$ is increasing in $L$ and $\epsilon$, both natural conclusions. Thus, aircraft quietness falls $(n$ rises) as the noise constraint is relaxed or the cost of abatement increases. In addition, (13) shows that a higher fixed cost per flight raises $n$, while an increase in the delay disutility has the opposite effect. All of these comparative-static results are summarized in Table 1.

These results show that tighter noise regulation under a cumulative constraint hurts airline 
passengers, with the impact operating through two channels. Flight frequency falls, reducing service quality, and fares rise, so that the cost of travel escalates as it convenience declines. For tighter regulation to be desirable, these losses must be offset by social gains from a lower noise level, as analyzed below. ${ }^{14}$

\subsection{Sequential choice under a cumulative constraint}

Using the simultaneous-choice case as a benchmark, consider now the case where frequencies and fares are chosen sequentially. Under sequential choice, fares are set conditional on frequencies, with frequencies then chosen in a first stage, taking into account the second-stage impact on fares.

To analyze this case, the first-order condition for choice of $p_{1}$ (eq. (9)) is supplemented with the analogous condition for airline 2, gotten by reversing the 1 and 2 subscripts in (9). The equations are then solved for $p_{1}$ and $p_{2}$ as functions of $f_{1}$ and $f_{2}$. The solution for $p_{1}$ is given by

$$
p_{1}=\alpha / 2+\tau-\frac{1}{3}\left(\frac{\gamma}{f_{1}}-\frac{\gamma}{f_{2}}+\frac{2 \epsilon f_{1}+\epsilon f_{2}}{L}\right),
$$

and the $p_{2}$ solution is gotten by reversing the 1 and 2 subscripts. These solutions are substituted into the profit function (8) for airline 1 , which is differentiated with respect to $f_{1}$ treating $f_{2}$ as parametric. Symmetry is imposed in the resulting first-order condition, which is then solved for $f .^{15}$ The result is

$$
f=\sqrt{\frac{\gamma}{3 \theta+\epsilon / L}},
$$

which differs from (12) by the presence of the factor 3, rather than 2, in the denominator. Thus, for a given $L$, frequency is smaller in the sequential-choice case. The $p$ solution in (11), which remains relevant, shows that the smaller $f$ leads to a lower fare under sequential choice. In addition, the lower $f$ leads to a smaller aircraft size, and aircraft are also noisier under sequential choice (the 2 factor in (13) is replaced by 3 ).

Despite these differences, the timing of airline decisions has no effect on the main comparative-static properties of the equilibrium. In particular, it is easy to see that the change in the $f$ solution leaves the signs in Table 1 unaffected, so that the table applies to both the 
sequential and simultaneous-choice cases. ${ }^{16}$

The key conclusions of the preceding analysis are then summarized as follows:

Proposition 1. A reduction in the allowed noise level under a cumulative noise constraint leads to lower flight frequency, larger and quieter aircraft, and a higher fare, regardless of whether airline choices are simultaneous or sequential. Airline passengers are hurt by the tighter noise limit.

\subsection{The case of a per-aircraft noise constraint}

Suppose now that noise regulation takes the form of a per-aircraft noise constraint, written as $n_{i} \leq \bar{n}, i=1,2$. Since the constraint will bind, airline 1's objective function is given by (8) with $n_{1}$ replaced by $\bar{n}$. Under simultaneous choice, the previous first-order condition (9) for $p_{1}$ remains relevant, but with $L$ replaced by $f_{1} \bar{n}$. Imposing symmetry, the fare solution is then

$$
p=\alpha / 2+\tau+\epsilon / \bar{n}
$$

which corresponds to the previous solution in (11) after substitution for $L$. This equivalence implies that, if $L$ under the cumulative constraint is set so that noise per aircraft in (13) is equal to $\bar{n}$, the same fare levels emerge under the two regimes.

The first-order condition for $f_{1}$ is now

$$
\frac{\partial \pi_{1}}{\partial f_{1}}=\frac{\gamma\left(p_{1}-\tau-\epsilon / \bar{n}\right)}{\alpha f_{1}^{2}}-\theta=0
$$

and, substituting (16), it yields a symmetric frequency solution of

$$
f=\sqrt{\frac{\gamma}{2 \theta}} .
$$

Under sequential choice, which is analyzed by following the same steps as before, the fare solution in (16) remains relevant but frequency is given by

$$
f=\sqrt{\frac{\gamma}{3 \theta}},
$$


where a factor of 3 replaces the 2 in the simultaneous solution, as in the cumulative case. ${ }^{17}$

Under both the simultaneous and sequential solutions, frequency is increasing in $\gamma$ and decreasing in $\theta$, as in the cumulative case. But frequency does not depend on $\bar{n}$ and is thus independent of the stringency of the noise constraint, in contrast to the cumulative case. With $f$ independent of the noise limit, $\bar{n} f$ and thus total noise $L$ rises as $\bar{n}$ is raised, and $L$ rises with $\gamma$ and falls with $\theta$, holding $\bar{n}$ fixed. In addition, since the fare solution in (16) does not involve $f, p$ is now independent of $\gamma$ and $\theta$, although the fare responds positively to $\alpha$ and $\tau$, as before. These comparative-static effects are summarized in Table 2. Airline passengers are hurt, as in the cumulative case, by a tighter noise constraint, but the effect now operates through a single channel, a higher fare, with service quality unchanged. ${ }^{18}$

As in the cumulative case, sequential choice yields a higher frequency and smaller aircraft size than simultaneous choice. But since $p$ is independent of $f$, fare levels are the same under the two choice scenarios, in contrast to the previous conclusion.

A more interesting observation, however, is that frequency is always higher under a peraircraft noise constraint than in the cumulative case, with the opposite conclusion applying to aircraft size. These results follow because the simultaneous frequency solution in (18) is larger than the corresponding cumulative solution (12), with the same comparison applying to the sequential solutions (19) and (15). Apparently, lower frequencies arise in the cumulative case because a reduction in $f$ provides a means of satisfying the cumulative constraint.

Summarizing the main conclusions of the above analysis yields

Proposition 2. (i) A reduction in the allowed noise level under a per-aircraft constraint raises the fare, harming airline passengers, while having no effect on flight frequency or aircraft size. Total noise falls.

(ii) Flight frequency is always higher and aircraft size lower under a per-aircraft noise constraint than under a cumulative constraint, but fares are identical in the two cases when the constraints are set to achieve the same noise per aircraft.

\subsection{The monopoly case}

In the monopoly case, analyzed by Girvin (2006a), different comparative-static results emerge. Although closed-form solutions for the decision variables are not available, her analysis 
shows that a tightening of the cumulative noise constraint reduces flight frequency, as in Proposition 1. A lower $L$ also affects traffic $q$, which no longer assumes the constant equilibrium value of $1 / 2$, causing it to drop. With $f$ and $q$ both falling, the change in aircraft size is ambiguous, and the same conclusion applies to aircraft quietness and the fare. Under a peraircraft noise constraint, a reduction in $\bar{n}$ lowers $f$, in contrast to Proposition 2 , while also reducing traffic. In addition, aircraft size falls. These conclusions show that the duopoly and monopoly equilibria have very different properties.

\section{Noise Taxation}

4.1. The equivalence of noise taxation and cumulative regulation

Under noise taxation, the airlines pay a tax of $t$ per unit of noise. ${ }^{19}$ As a result, the additional term $t f_{1} n_{1}$ is subtracted in the profit expression (8), which becomes

$$
\pi_{1}=\left(p_{1}-\tau-\epsilon / n_{1}\right)\left(\frac{1}{2}-\frac{1}{\alpha}\left[p_{1}-p_{2}+\gamma / f_{1}-\gamma / f_{2}\right]\right)-\theta f_{1}-t f_{1} n_{1}
$$

With simultaneous choice, this expression is maximized by choice of $p_{1}, f_{1}$, and $n_{1}$ without the presence of any constraint. The first-order condition for $p_{1}$ reduces to the previous condition (16), with $\bar{n}$ replaced by $n_{1}$. After imposing symmetry, the first-order conditions for $f_{1}$ and $n_{1}$ reduce, respectively, to:

$$
\begin{aligned}
n f & =\frac{\epsilon}{2 t n} \\
f^{2} & =\frac{\gamma}{2 \theta+2 t n}
\end{aligned}
$$

These conditions determine the symmetric equilibrium values of $f$ and $n$ conditional on $t$. To compare the outcome to the other regimes, it is useful to change variables so that (21) and (22) are expressed in terms of $f$ and $L$, where $L$ is endogenous. Using (21) along with $n f=L$ to eliminate $2 t n,(22)$ becomes

$$
f=\sqrt{\frac{\gamma}{2 \theta+\epsilon / L}},
$$


which is the same as the cumulative frequency solution (12) under simultaneous choice. In addition, since $(21)$ can be rewritten as $(n f)^{2} / f=\epsilon / 2 t$, the condition becomes

$$
-\frac{\epsilon f}{L^{2}}+2 t=0
$$

Substituting (23) into (24) and simplifying yields the following condition that determines $L$ as a function of $t$ :

$$
\sqrt{\frac{\gamma \epsilon}{8 \theta L^{4} / \epsilon+4 L^{3}}}=t
$$

To obtain the entire solution under noise taxation for a given $t$, (25) is solved for the corresponding $L$, and substitution in (23) then yields $f$ and consequently $n$. The fare solution in (16) then yields $p$.

Because (23) is the same as the cumulative frequency solution, a key implication of (23) and (25) is that noise taxation is equivalent to cumulative noise regulation. In other words, the outcome generated by a particular cumulative noise limit $L^{\#}$ can be exactly replicated under noise taxation. To do so, $L^{\#}$ is plugged into (25), and $t$ is set at the resulting value, denoted $t^{\#}$. When the airlines maximize facing $t^{\#}$, a total noise level of $L^{\#}$ per carrier is generated endogenously, and the $f, n$ and $p$ values corresponding to the cumulative limit then emerge.

The same conclusion emerges under sequential choice. Fares are again chosen in a second stage, while airline 1 chooses $f_{1}$ and $n_{1}$ in the first stage taking the second-stage fare impacts into account and viewing $f_{2}$ and $n_{2}$ as parametric. Given this behavior, a lengthy derivation yields symmetric first-order conditions with the same form as (21) and (22). The difference is that a factor of 3 replaces the 2 in each instance in these equations. ${ }^{20}$ As a result, 3 replaces 2 in (23) and (24), and 18 replaces 8 in (25). Since the modified (23) is then the same as the sequential solution in (15), the same argument as before establishes that noise taxation is equivalent to cumulative noise regulation under sequential choice. In other words, the cumulative outcome for a given $L=L^{\#}$ can be exactly replicated under noise taxation by a suitable choice of $t$. Summarizing yields ${ }^{21}$

Proposition 3. Noise taxation and cumulative noise regulation are equivalent, regardless of whether airline choices are simultaneous or sequential. In particular, the 
outcome under cumulative regulation can be exactly replicated by suitable choice of the noise tax $t$.

The apparent explanation for this result is that both the tax and cumulative regimes give the airline the same margins of adjustment $(f$ and $n)$, even though these variables must satisfy a constraint in the cumulative case. In the per-aircraft regime, by contrast, the airline's adjustment margins are limited.

\subsection{Comparative statics under noise taxation}

Inspection of (25) shows how parameter changes affect $L$. First, since the LHS is decreasing in $L$, if follows that a higher $t$ reduces total noise per carrier, a natural result. In addition, it is easily seen that $L$ is decreasing in $\theta$ and increasing in both $\gamma$ and $\epsilon$, while being independent of $\alpha$ and $\tau$. Taking account of the effects on $L$, inspection of (23) shows that $f$ is decreasing in $t$ and $\theta$ and increasing in $\gamma$, while an additional argument shows that $f$ decreases with $\epsilon$. Since $n=\epsilon / 2 L t$ from (21), eliminating $t$ using (25) yields $n=\sqrt{\left(2 \theta L^{2}+\epsilon L\right) / \gamma}$. Using the impacts on $L$, it follows from the latter solution that $n$ is decreasing in $t$ and increasing in $\epsilon$. Again using impacts on $L$, the first solution shows that $n$ is decreasing in $\gamma$ and increasing in $\theta$. Finally, using (16) with $n$ in place of $\bar{n}$, inspection shows that $p$ is increasing in $t$ and decreasing in $\gamma$ and $\theta$, and an additional argument shows that $p$ increases with $\epsilon .^{22}$

These results are summarized in Table 3. Note that the comparative-static effects of $\epsilon, \tau$, $\alpha, \gamma$, and $\theta$ are naturally the same as in the cumulative case (compare the last five columns of Tables 1 and 3). Finally, observe that since $f$ falls and $p$ rises with $t$, an increase in the noise tax hurts airline passengers. These results, which also apply to the sequential case, are summarized as follows

Proposition 4. An increase in the noise tax leads to less total noise, lower flight frequency, larger and quieter aircraft, and a higher fare, regardless of whether airline choices are simultaneous or sequential. Airline passengers are hurt by the higher noise $\operatorname{tax}$. 


\section{Welfare Analysis}

This section of the paper carries out welfare analysis. ${ }^{23}$ Social welfare in the model is equal to consumer surplus plus airline profit minus environmental noise damage. Since the fare paid is just a transfer between consumers and the airlines, while the benefits from travel and brand loyalty are constants, the planner can focus on a welfare measure equal to airline costs plus the cost of consumer schedule delay plus noise damage. Imposing symmetry on the airlines, and writing welfare in terms of $L$, the welfare expression equals

$$
W=\tau+2 \theta f+\frac{\epsilon f}{L}+\frac{\gamma}{f}+\lambda 2 L
$$

where noise damage is assumed for simplicity to be a linear function of total noise (equal to $2 L)$, with parameter $\lambda .^{24}$ To interpret the rest of (26), recall that total traffic is equal to unity, which then multiplies the marginal seat $\operatorname{cost} \tau$ and schedule delay cost, $\gamma / f$. With two airlines, fixed cost acquires a factor of 2 , and total noise abatement cost is $2 f(\epsilon s / n)=\epsilon / n=\epsilon f / L$, where the first equality uses the fact that $2 f s$ equals the total unitary traffic level. The planner's goal is to minimize (26) by choice of $f$ and $L$. Equivalently, (26) could be rewritten in terms of $n$, which would be chosen optimally along with $f .^{25}$

Note that, because the fare cancels in computing $W$, the impacts of noise regulation on passengers that operate through $p$ are irrelevant in the welfare analysis. Only those effects operating through schedule-delay costs matter.

\subsection{The first-best optimum and the second-best optimum under a cumulative constraint}

Viewing $L$ as the choice variable, it is useful to derive the social optimum in two steps. First, $f$ is chosen to minimize (26) conditional on $L$, yielding the first-order condition

$$
2 \theta+\frac{\epsilon}{L}-\frac{\gamma}{f^{2}}=0
$$

and the resulting frequency solution

$$
f^{*}(L) \equiv \sqrt{\frac{\gamma}{2 \theta+\epsilon / L}},
$$


where $f^{*}(L)$ denotes the optimal frequency conditional on $L$. Next, (28) is substituted back into (26), which is differentiated with respect to $L$. Using the envelope theorem, the resulting first-order condition is

$$
-\frac{\epsilon f^{*}(L)}{L^{2}}+2 \lambda=0,
$$

which says that the extra noise damage should equal the saving in abatement cost when $L$ is marginally increased. After substituting (28) into (29) and simplifying, the socially optimal noise level, denoted $L^{*}$ and referred to as the first-best optimal value, turns out to be the root of a 4th degree polynomial in $L$. The first-best optimal $f$ then equals $f^{*}\left(L^{*}\right)$, and the optimal $n$ is given by $n^{*}=L^{*} / f^{*}\left(L^{*}\right)$.

The conditionally optimal $f$ solution in (28) reveals the efficiency of airline choices under a cumulative constraint, for a given value of $L$. Since (28) and (12) are the same, it follows that for a given $L$, the airline's frequency choice is efficient, provided that its choices are made in simultaneous fashion. However, if choice is sequential, then the airline's chosen frequency is lower than the one desired by the planner, as seen by comparing (15) and (28), and aircraft size is correspondingly too large.

With the airline's choices efficient under simultaneous choice, it follows that the planner could set $L$ at $L^{*}$, allow the airline to maximize profit, and be assured that the outcome would be socially optimal. However, under more-realistic sequential behavior, the airline makes the wrong choices conditional on $L$, which means that setting $L$ at $L^{*}$ would fail to generate the social optimum. Thus, in the sequential case, the planner must take the airline's distorted choices into account in setting $L$, which leads to the second-best optimal level under sequential choice, denoted $\hat{L}$.

To characterize $\hat{L}$, let $\hat{f}(L)$ denote the airline's profit-maximizing sequential frequency choice conditional on $L$, given by (15). With the planner obliged to accept airline choices, his objective function comes from substituting $\hat{f}(L)$ into (26), which yields

$$
W=\tau+2 \theta \hat{f}(L)+\frac{\epsilon \hat{f}(L)}{L}+\frac{\gamma}{\hat{f}(L)}+\lambda 2 L .
$$


Differentiating (30), $\hat{L}$ must satisfy

$$
-\frac{\epsilon \hat{f}(L)}{L^{2}}-\left(\frac{\gamma}{\hat{f}(L)^{2}}-2 \theta-\frac{\epsilon}{L}\right) \hat{f}^{\prime}(L)+2 \lambda=0 .
$$

Since the term in parentheses in (31) reduces to $\theta$ using (15), the condition can be written

$$
-\frac{\epsilon \hat{f}(L)}{L^{2}}-\theta \hat{f}^{\prime}(L)+2 \lambda=0 .
$$

Substituting $\hat{f}(L)$ and simplifying, (32) reduces to a 6th degree polynomial equation in $L$, which determines $\hat{L}$, the second-best optimal value.

An interesting question is whether $\hat{L}$ is larger or smaller than the first-best optimal value $L^{*}$. In other words, when the planner must accept the airline's inefficient choices, does he set a cumulative noise limit looser or tighter than the first-best level? To answer this question, (32) must be compared to the first-best condition in (29). As functions of $L$, both LHS expressions must be upward-sloping curves at the respective optima to ensure minimization of $W$, which means that the higher curve is associated with the smaller $L$ solution. On the one hand, the presence of the term $-\theta \hat{f}^{\prime}(L)$, which is negative by Proposition 1, tends to make the secondbest curve from (32) lower than the first-best curve, which in turn tends to make $\hat{L}$ larger than the $L^{*}$. The intuitive explanation comes from recalling that the conditional sequential frequency solution is inefficiently low, with $\hat{f}(L)<f^{*}(L)$. Given $\hat{f}^{\prime}(L)>0$, it follows that the planner can use his second-best $L$ choice to raise $f$ from its inefficiently low level, an intervention that would require setting $L$ above its first-best value. On the other hand, the fact that $\hat{f}(L)<f^{*}(L)$ makes the initial term in the second-best expression from (32) larger (less negative) than the corresponding term in the above first-best expression, which tends to make the second-best curve higher than the first-best curve.

Despite this apparent indeterminacy, once the $f^{*}$ and $\hat{f}$ functions are substituted into (29) and (32) and appropriate manipulations are made, it can be shown that $\hat{L}<L^{*}$ holds (see the appendix). This conclusion means that the latter effect discussed above (which depresses $\hat{L}$ ) dominates the goal of using $\hat{L}$ to boost the airlines' inefficiently low flight frequency. With 
$\hat{L}<L^{*}$, it follows from (15) and $(28)$ that $\hat{f}(\hat{L})<f^{*}\left(L^{*}\right)$, so that the second-best flight frequency under sequential choice is inefficiently low. In addition, using $\hat{L}<L^{*}$, it can be shown that $n^{*}<\hat{n}=\hat{L} / \hat{f}(\hat{L})$, where $\hat{n}$ is the second-best optimal $n$ value (see the appendix). Summarizing yields ${ }^{26}$

Proposition 5. The second-best cumulative noise limit under sequential choice is less than the first-best limit $\left(\hat{L}<L^{*}\right)$. In addition, the associated second-best flight frequency is lower than the first-best level, and the second-best per-aircraft noise level is higher than the first-best level.

\subsection{What noise-tax levels support the optima?}

Since noise taxation and cumulative noise regulation are equivalent, it follows that the above optima can be generated by suitable selection of the noise tax $t$. Consider first the case where airlines make simultaneous choices. As seen above, when $L$ is set at $L^{*}$ under cumulative regulation, airline decisions yield the first-best optimum. But since any outcome under cumulative regulation can be generated by an appropriate noise tax, it follows that a particular value of the tax yields the first-best optimum.

To derive that value, observe that (23) and (24) jointly determine the solutions for $f$ and $L$ under a particular level of the noise tax $t$. But this equation system has the same form as (28) and (29), which determine the first-best optimum. The two systems can be made identical, ensuring that the noise-tax solution coincides with the social optimum, when $t$ is set at the appropriate value, denoted $t^{*}$. As can be seen, the correct value is $t^{*}=\lambda$, indicating that the noise tax should be set equal to the marginal noise damage, a natural conclusion.

Suppose instead that airline choices are sequential, so that the second-best optimum is relevant. To find the noise tax that generates this optimum, recall that the solution under noise taxation is given by (15) along with the modified version of (24), which is written $-\epsilon f / L^{2}+3 t=$ 0 . Thus, the equation determining $L$ under noise taxation can be written $-\epsilon \hat{f}(L) / L^{2}+3 t=0$. For the resulting solution to coincide with the second-best optimum, $t$ must be set at the value $\hat{t}$ satisfying

$$
-\frac{\epsilon \hat{f}(\hat{L})}{\hat{L}^{2}}+3 \hat{t}=0 .
$$


But since (32) must hold at $L=\hat{L},(33)$ can be used to eliminate the first term, allowing the equation to be rewritten as

$$
-3 \hat{t}-\theta \hat{f}^{\prime}(\hat{L})+2 \lambda=0
$$

Rearranging then yields

$$
\hat{t}=\frac{2}{3} \lambda-\frac{1}{3} \theta \hat{f}^{\prime}(\hat{L})<\lambda \equiv t^{*}
$$

(note that (32) implies positivity of $\hat{t}$ ). Thus, the noise tax rate that generates the second-best optimum under cumulative regulation is less than the marginal noise damage and thus less than $t^{*}$. Note that this conclusion might seem inconsistent with the inequality $\hat{L}<L^{*}$ from Proposition 5 given that a low tax should be associated with a large, not a small, $L$. But since this inverse relationship holds airline choice behavior fixed, while $\hat{t}$ and $t^{*}$ apply under different behaviors (simultaneous vs. sequential choice), no inconsistency arises.

It is important to recognize that, since noise taxation and cumulative noise regulation are equivalent under sequential choice, and since $\hat{L}$ is the best possible value of $L$, a tax rate different from $\hat{t}$ (one associated with a different value of $L$ ) cannot yield a higher welfare level. Thus, $\hat{t}$ is the welfare maximizing noise tax rate under sequential choice, and it is less than the marginal noise damage $\lambda$. Summarizing yields

Proposition 6. If airline choices are simultaneous, the first-best optimum can be generated by a noise tax equal to the marginal noise damage $\lambda$. If airline choices are instead sequential, the welfare maximizing tax rate is less than $\lambda$, and it generates the second-best optimum under cumulative regulation.

\subsection{Could per-aircraft noise regulation be superior?}

When airline choices are simultaneous, per-aircraft regulation can never be superior to cumulative regulation (or equivalent noise taxation) since these regimes can generate the firstbest optimum. Per-aircraft regulation is, by contrast, inefficient given that the simultaneous frequency solution in (18) is always larger than the first-best optimal level, $f^{*}\left(L^{*}\right)$. But when choices are sequential, this conclusion can be overturned. To understand this claim, consider 
the following inequalities, where $\tilde{f}$ denotes the sequential frequency solution from (19) under per-aircraft regulation:

$$
\tilde{f} \equiv \sqrt{\frac{\gamma}{3 \theta}}>\sqrt{\frac{\gamma}{3 \theta+\epsilon / \hat{L}}} \equiv \hat{f}(\hat{L})<f^{*}\left(L^{*}\right)
$$

Thus, $\tilde{f}$ is larger than $\hat{f}(\hat{L})$, the second-best optimal frequency under cumulative regulation, which in turn is smaller than the first-best optimal frequency $f^{*}\left(L^{*}\right)$ by Proposition 5 . Given (36), $\tilde{f}$ could be closer to $f^{*}\left(L^{*}\right)$ than is $\hat{f}(\hat{L})$, raising the possibility that per-aircraft regulation is superior to cumulative regulation.

Since welfare under the per-aircraft regime also depends on the magnitude of $n$, exploring this question requires computation of the second-best $\bar{n}$ under sequential choice. To do so, the welfare expression in (26) is rewritten in terms of $\bar{n}$ and $\tilde{f}$ is then substituted, so that the last four terms become $2 \theta \tilde{f}+\epsilon / \bar{n}+\gamma / \tilde{f}+\lambda 2 \bar{n} \tilde{f}$. The second-best optimal per-aircraft noise limit is found by differentiating this expression with respect to $\bar{n}$, noting that $\tilde{f}$ from $(36)$ is independent of $\bar{n}$. The resulting first-order condition is

$$
-\frac{\epsilon}{\bar{n}^{2}}+2 \lambda \tilde{f}=0
$$

After using (36) to substitute for $\tilde{f}$, (37) can be solved for $\bar{n}$ and the result substituted into the above welfare expression. If per-aircraft regulation is superior, the resulting welfare level is higher than the one achieved under cumulative regulation.

This comparison cannot be carried out analytically, but illustrative numerical examples can be computed. In generating the examples, the marginal-cost parameter $\tau$ is normalized to one, while four other parameters are allowed to vary. ${ }^{27}$ The delay-disutility and fixed-cost parameters $\gamma$ and $\theta$ vary between 0.1 and 10.1 in steps of 1 , while three combinations of the abatement-cost and noise damage parameters are considered: $(\epsilon, \lambda)=(1,1),(10,1)$, and $(10,10)$. Since the model is highly stylized, realistic calibration is not possible, making these parameter choices arbitrary. For each parameter combination, the polynomial equations for the various optima have only one positive solution, and the curves are upward sloping at the solution values, ensuring that these values represent minima for $W$. 
The welfare comparison between the two regulatory regimes, given as ratios of the cumulative and per-aircraft welfare levels, is shown in Table 4. The results show that welfare under the per-aircraft regime tends to be larger than under the cumulative regime when $\gamma$ and $\theta$ are big and smaller otherwise, with the margin of reversal shifting outward as $\epsilon$ and $\lambda$ assume larger values. Note that in the third panel of Table 4, welfare is higher under the cumulative regime for all the values of $\gamma$ and $\theta$. Summarizing yields

Proposition 7. When airline choices are sequential, the second-best welfare level may be higher under per-aircraft noise regulation than under cumulative regulation or the equivalent tax regime.

Despite this conclusion, further inspection of Table 4 reveals that the choice between the two regulatory regimes is actually a matter of indifference for most parameter values, with the ratios usually very close to 1 . However, when $\gamma$ and $\theta$ are both small, the per-aircraft regime becomes markedly inferior to the cumulative regime. With the regimes otherwise close to equivalent, this finding suggests that cumulative noise regulation (or equivalent noise taxation) may be preferable to per-aircraft regulation. Of course, this conclusion might be overturned by results from other unexplored regions of the parameter space.

A further numerical observation concerns the magnitude of the welfare sacrifice from the planner's inability to implement the first-best optimum. Interestingly, this sacrifice is very small: second-best welfare under the cumulative regime is never smaller than 98 percent of the first-best level, and the per-aircraft regime yields a similarly good outcome over most of the parameter region. But for those parameter values where the per-aircraft regime falls well short of the cumulative regime, the loss it generates relative to the first-best optimum is similarly large. $^{28}$

\section{Conclusion}

This paper has explored the impact of airport noise regulation on airline service quality and airfares. It has also characterized the socially optimal stringency of noise limits, taking both noise damage and the various costs borne by airlines and their passengers into account, while analyzing the use of noise taxes. Along with the companion paper by Girvin (2006a), this 
work represents the first complete theoretical investigation into the economics of airport noise regulation using a model where the interests of the key relevant stakeholders are captured.

The main conclusions of the analysis are as follows: (i) noise regulation harms airline passengers by raising fares and potentially reducing service quality; (ii) cumulative and per-aircraft noise regulation have quite different effects on airline decisions; (iii) cumulative regulation appears to be superior from a social-welfare perspective; (iv) under realistic sequential airline choice behavior, the best a planner can do under cumulative regulation is to use an inefficiently tight noise limit that yields lower-than-optimal flight frequency; (iv) noise taxation is equivalent to cumulative noise regulation, generating exactly the same airline decisions when the tax rate is suitably chosen.

The analysis has treated noise taxes and the two noise-regulation regimes, cumulative and per-aircraft, as alternatives, when in fact these approaches often coexist in actual practice. In other words, the FAA certification standard puts an upper bound on noise per aircraft, while individual airports impose cumulative noise limits and other operating constraints, including noise taxes. Given this real-world complexity, what practical lessons can be drawn from the analysis? To reach an answer, the first observation is that most aircraft currently in production already have lower noise signatures than the latest Stage 4 noise limits. ${ }^{29}$ This fact, along with the presence of additional airport-level regulations, apparently means that airports do not find the existing per-aircraft noise limits to be sufficiently stringent. Therefore, they impose additional regulations, which generate a demand for aircraft quieter than the current FAA standard.

Instead of allowing aircraft quietness to be effectively determined by airport-level policies, the FAA could instead impose a per-aircraft limit that is even more stringent than the Stage 4 limit. But since a cumulative noise limit (or the equivalent tax) appears to be a superior instrument, the analysis suggests that airport-level regulation may actually be a preferable policy.

Although the highly stylized framework presented in this paper captures the essential elements of the airport noise problem, additional refinements and extensions of the model could be beneficial. For example, more-realistic specifications of the airline's noise-abatement 
cost function and the functions giving cumulative noise and noise damage would improve the model's accuracy. Moreover, adding the airport itself to the list of stakeholders in the model could provide further insights. The airport, responding to political pressure to reduce noise, could be portrayed as choosing the noise regulatory regime that maximizes its profit for a given target noise level. In other extensions, concurrent implementation of noise-abatement measures, such as a nighttime curfew and a noise tax, could be modeled, and airline asymmetry (with one large and one small carrier) could be introduced. Analysis of these extended models might, in some cases, require numerical methods. But the insights gained from numerical models, especially those that can be calibrated with empirical data, might prove valuable in guiding airport noise policies. 


\section{Appendix}

Proof of Proposition 5:

Combining (29) and (32), $L^{*}$ and $\hat{L}$ must satisfy

$$
\frac{\epsilon \hat{f}(\hat{L})}{\hat{L}^{2}}+\theta \hat{f}^{\prime}(\hat{L})=\frac{\epsilon f^{*}\left(L^{*}\right)}{L^{* 2}}
$$

Substituting for the $f^{*}$ and $\hat{f}$ functions using (15) and (28), (a1) becomes

$$
\frac{\epsilon}{\hat{L}^{2}} \sqrt{\frac{\gamma}{3 \theta+\epsilon / \hat{L}}}+\frac{\theta \epsilon}{2 \hat{L}^{2}(3 \theta+\epsilon / \hat{L})} \sqrt{\frac{\gamma}{3 \theta+\epsilon / \hat{L}}}=\frac{\epsilon}{L^{*} 2} \sqrt{\frac{\gamma}{2 \theta+\epsilon / L^{*}}}
$$

Rearranging and squaring both sides, (a2) reduces to

$$
\frac{2 \theta L^{*}+\epsilon}{3 \theta \hat{L}+\epsilon}\left[\frac{7 \theta \hat{L}+2 \epsilon}{6 \theta \hat{L}+2 \epsilon}\right]^{2}=\left[\frac{\hat{L}}{L^{*}}\right]^{3}
$$

Suppose that $\hat{L} \geq L^{*}$ holds. Then the LHS of (a3) must exceed 1. But after rearranging, the resulting inequality reduces to

$$
L^{*} \geq \hat{L}\left[\frac{108 \theta^{3} \hat{L}^{2}+59 \theta^{2} \epsilon \hat{L}+8 \theta \epsilon^{2}}{98 \theta^{3} \hat{L}^{2}+56 \theta^{2} \epsilon \hat{L}+8 \theta \epsilon^{2}}\right] .
$$

Since the ratio term in $(a 4)$ exceeds 1 , the inequality implies $L^{*}>\hat{L}$, which contradicts the initial assumption that $\hat{L} \geq L^{*}$, implying that $\hat{L}<L^{*}$ must hold instead.

To establish $n^{*}<\hat{n}$, note that $(a 1)$ can be rewritten as

$$
\frac{\epsilon}{\hat{n} \hat{L}}+\theta \hat{f}^{\prime}(\hat{L})=\frac{\epsilon}{n^{*} L^{*}}
$$

which implies $\hat{n} \hat{L}>n^{*} L^{*}$. The conclusion follows given $\hat{L}<L^{*}$. 


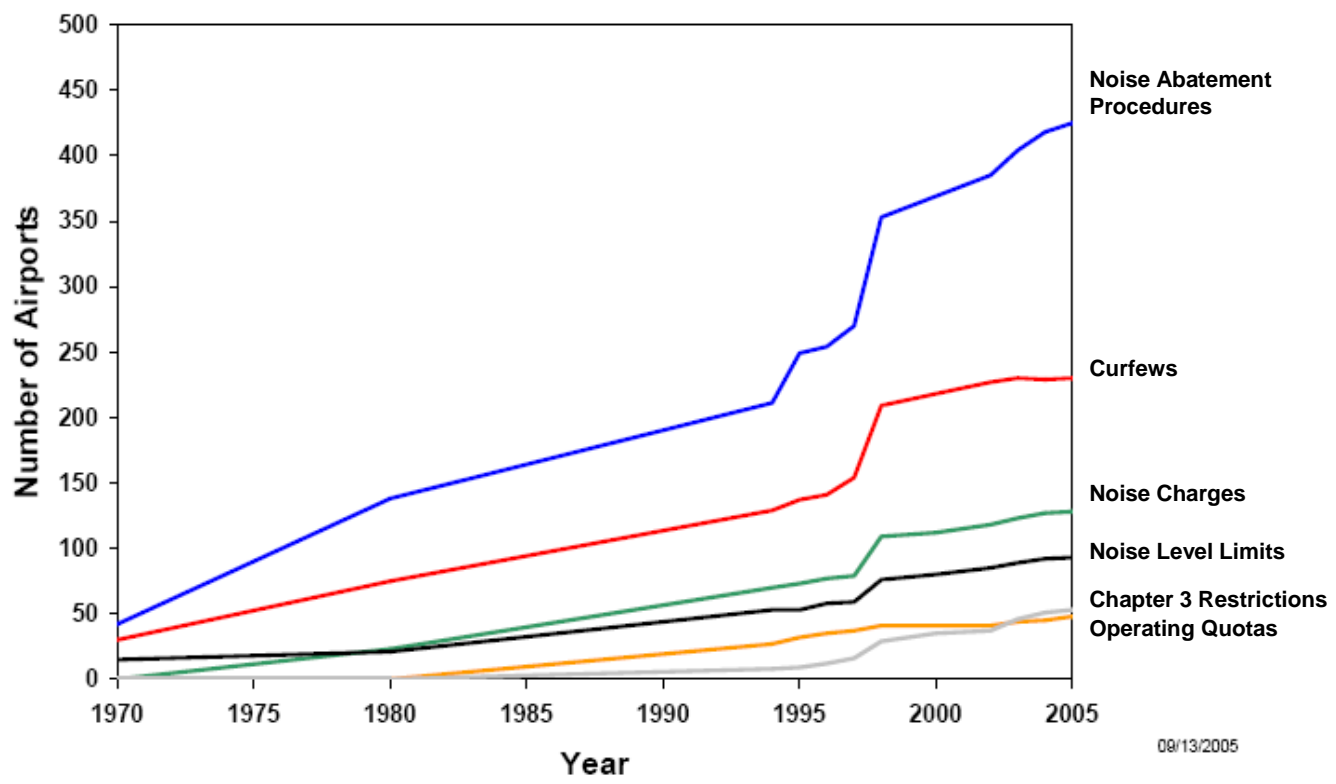

Figure 1. Trends in Airport Noise Restrictions

(Source: http://www.boeing.com/commercial/noise/airports2005.pdf) 
Table 1.

Comparative-Static Effects Under a Cumulative Constraint

\begin{tabular}{ccccccc} 
& $L$ & $\epsilon$ & $\tau$ & $\alpha$ & $\gamma$ & $\theta$ \\
\hline \hline$f$ & + & - & 0 & 0 & + & - \\
$n$ & + & + & 0 & 0 & - & + \\
$p$ & - & + & + & + & + & - \\
$s$ & - & + & 0 & 0 & - & + \\
\hline \hline
\end{tabular}

Table 2.

Comparative-Static Effects Under a Per-Aircraft Constraint

\begin{tabular}{ccccccc} 
& $n$ & $\epsilon$ & $\tau$ & $\alpha$ & $\gamma$ & $\theta$ \\
\hline \hline$L$ & + & 0 & 0 & 0 & + & + \\
$f$ & 0 & 0 & 0 & 0 & + & + \\
$p$ & - & + & + & + & 0 & 0 \\
$s$ & 0 & 0 & 0 & 0 & - & + \\
\hline \hline
\end{tabular}

Table 3.

Comparative-Static Effects Under Noise Taxation

\begin{tabular}{ccccccc} 
& $t$ & $\epsilon$ & $\tau$ & $\alpha$ & $\gamma$ & $\theta$ \\
\hline \hline$L$ & - & + & 0 & 0 & + & - \\
$f$ & - & - & 0 & 0 & + & - \\
$n$ & - & + & 0 & 0 & - & + \\
$p$ & + & + & + & + & + & - \\
$s$ & + & + & 0 & 0 & - & + \\
\hline \hline
\end{tabular}


Table 4.

Ratio of Second-Best Welfare under Cumulative Noise Limit to Welfare under Per-Aircraft Noise Limit

\begin{tabular}{|c|c|c|c|c|c|c|c|c|c|c|c|}
\hline & & & & & & 1) & & & & & \\
\hline$\gamma$ & 0.1 & 1.1 & 2.1 & 3.1 & 4.1 & 5.1 & 6.1 & 7.1 & 8.1 & 9.1 & 10.1 \\
\hline 0.1 & 1.3634 & 1.0276 & 1.0028 & 0.9954 & 0.9924 & 0.9911 & 0.9905 & 0.9903 & 0.9903 & 0.9904 & 0.9905 \\
\hline 1.1 & 1.1983 & 0.9994 & 0.9915 & 0.9903 & 0.9904 & 0.9908 & 0.9912 & 0.9917 & 0.9921 & 0.9925 & 0.9928 \\
\hline 2.1 & 1.1638 & 0.9957 & 0.9906 & 0.9903 & 0.9908 & 0.9913 & 0.9919 & 0.9924 & 0.9928 & 0.9932 & 0.9936 \\
\hline 3.1 & 1.1449 & 0.9940 & 0.9904 & 0.9905 & 0.9911 & 0.9917 & 0.9923 & 0.9928 & 0.9933 & 0.9936 & 0.9940 \\
\hline 4.1 & 1.1322 & 0.9930 & 0.9903 & 0.9907 & 0.9914 & 0.9920 & 0.9926 & 0.9931 & 0.9936 & 0.9939 & 0.9943 \\
\hline 5.1 & 1.1228 & 0.9924 & 0.9903 & 0.9908 & 0.9916 & 0.9923 & 0.9929 & 0.9934 & 0.9938 & 0.9942 & 0.9945 \\
\hline 6.1 & 1.1154 & 0.9920 & 0.9903 & 0.9910 & 0.9918 & 0.9925 & 0.9931 & 0.9936 & 0.9940 & 0.9944 & 0.9947 \\
\hline 7.1 & 1.1093 & 0.9917 & 0.9903 & 0.9911 & 0.9919 & 0.9926 & 0.9932 & 0.9937 & 0.9942 & 0.9945 & 0.9948 \\
\hline 8.1 & 1.1042 & 0.9914 & 0.9904 & 0.9912 & 0.9921 & 0.9928 & 0.9934 & 0.9939 & 0.9943 & 0.9947 & 0.9950 \\
\hline 9.1 & 1.0999 & 0.9912 & 0.9904 & 0.9913 & 0.9922 & 0.9929 & 0.9935 & 0.9940 & 0.9944 & 0.9948 & 0.9951 \\
\hline 0.1 & 1.0960 & 0.9910 & 0.9905 & 0.9914 & 0.9923 & 0.9930 & 0.9936 & 0.9941 & 0.9945 & 0.9949 & 0.9952 \\
\hline
\end{tabular}

\begin{tabular}{|c|c|c|c|c|c|c|c|c|c|c|c|}
\hline & & & & & $(\varepsilon=1$ & $=1)$ & & & & & 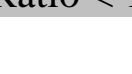 \\
\hline & $\theta$ & & & & & & & & & & \\
\hline$\gamma$ & 0.1 & 1.1 & 2.1 & 3.1 & 4.1 & 5.1 & 6.1 & 7.1 & 8.1 & 9.1 & 10.1 \\
\hline 0.1 & 1.8666 & 1.1877 & 1.0992 & 1.0615 & 1.0407 & 1.0277 & 1.0189 & 1.0127 & 1.0081 & 1.0046 & 1.0020 \\
\hline 1.1 & 1.5717 & 1.0831 & 1.0326 & 1.0138 & 1.0046 & 0.9994 & 0.9962 & 0.9942 & 0.9928 & 0.9919 & 0.9913 \\
\hline 2.1 & 1.5047 & 1.0633 & 1.0213 & 1.0064 & 0.9994 & 0.9957 & 0.9935 & 0.9922 & 0.9913 & 0.9908 & 0.9905 \\
\hline 3.1 & 1.4668 & 1.0528 & 1.0156 & 1.0029 & 0.9970 & 0.9940 & 0.9923 & 0.9914 & 0.9908 & 0.9905 & 0.9903 \\
\hline 4.1 & 1.4407 & 1.0460 & 1.0120 & 1.0006 & 0.9956 & 0.9931 & 0.9917 & 0.9909 & 0.9905 & 0.9903 & 0.9903 \\
\hline 5.1 & 1.4209 & 1.0410 & 1.0094 & 0.9991 & 0.9946 & 0.9924 & 0.9913 & 0.9907 & 0.9904 & 0.9903 & 0.9903 \\
\hline 6.1 & 1.4051 & 1.0372 & 1.0075 & 0.9980 & 0.9939 & 0.9920 & 0.9910 & 0.9905 & 0.9903 & 0.9903 & 0.9903 \\
\hline 7.1 & 1.3920 & 1.0341 & 1.0059 & 0.9971 & 0.9934 & 0.9917 & 0.9908 & 0.9904 & 0.9903 & 0.9903 & 0.9904 \\
\hline 8.1 & 1.3808 & 1.0315 & 1.0047 & 0.9964 & 0.9930 & 0.9914 & 0.9907 & 0.9904 & 0.9903 & 0.9903 & 0.9904 \\
\hline 9.1 & 1.3711 & 1.0293 & 1.0036 & 0.9958 & 0.9926 & 0.9912 & 0.9906 & 0.9903 & 0.9903 & 0.9904 & 0.9905 \\
\hline 10.1 & 1.3626 & 1.0275 & 1.0027 & 0.9953 & 0.9924 & 0.9911 & 0.9905 & 0.9903 & 0.9903 & 0.9904 & 0.9906 \\
\hline
\end{tabular}




\begin{tabular}{|c|c|c|c|c|c|c|c|c|c|c|c|}
\hline \multicolumn{12}{|c|}{$(\varepsilon=10, \lambda=10)$} \\
\hline & $\theta$ & & & & & & & & & & \\
\hline$\gamma$ & 0.1 & 1.1 & 2.1 & 3.1 & 4.1 & 5.1 & 6.1 & 7.1 & 8.1 & 9.1 & 10.1 \\
\hline 0.1 & 2.6722 & 1.5514 & 1.3697 & 1.2807 & 1.2258 & 1.1879 & 1.1599 & 1.1383 & 1.1211 & 1.1070 & 1.0953 \\
\hline 1.1 & 2.2096 & 1.3330 & 1.2027 & 1.1423 & 1.1068 & 1.0832 & 1.0665 & 1.0540 & 1.0444 & 1.0367 & 1.0306 \\
\hline 2.1 & 2.1014 & 1.2852 & 1.1677 & 1.1144 & 1.0835 & 1.0634 & 1.0492 & 1.0388 & 1.0309 & 1.0246 & 1.0196 \\
\hline 3.1 & 2.0394 & 1.2585 & 1.1485 & 1.0993 & 1.0711 & 1.0529 & 1.0402 & 1.0310 & 1.0240 & 1.0185 & 1.0142 \\
\hline 4.1 & 1.9963 & 1.2404 & 1.1356 & 1.0893 & 1.0630 & 1.0461 & 1.0344 & 1.0259 & 1.0195 & 1.0146 & 1.0107 \\
\hline 5.1 & 1.9635 & 1.2267 & 1.1261 & 1.0819 & 1.0570 & 1.0411 & 1.0302 & 1.0223 & 1.0164 & 1.0118 & 1.0082 \\
\hline 6.1 & 1.9371 & 1.2159 & 1.1185 & 1.0761 & 1.0523 & 1.0372 & 1.0269 & 1.0195 & 1.0140 & 1.0097 & 1.0064 \\
\hline 7.1 & 1.9151 & 1.2070 & 1.1124 & 1.0714 & 1.0486 & 1.0342 & 1.0243 & 1.0173 & 1.0121 & 1.0081 & 1.0049 \\
\hline 8.1 & 1.8962 & 1.1994 & 1.1072 & 1.0675 & 1.0454 & 1.0316 & 1.0222 & 1.0155 & 1.0105 & 1.0067 & 1.0037 \\
\hline 9.1 & 1.8798 & 1.1929 & 1.1027 & 1.0641 & 1.0428 & 1.0294 & 1.0204 & 1.0139 & 1.0092 & 1.0055 & 1.0027 \\
\hline 10.1 & 1.8652 & 1.1871 & 1.0988 & 1.0612 & 1.0405 & 1.0275 & 1.0188 & 1.0126 & 1.0080 & 1.0046 & 1.0019 \\
\hline
\end{tabular}




\section{References}

Brueckner, J.K., 2004. Network structure and airline scheduling, Journal of Industrial Economics 52, 291-311.

Brueckner, J.K, Flores-Fillol, R., 2006. Airline schedule competition. Unpublished paper, University of California, Irvine.

City of Long Beach, Airport Noise Compatibility Ordinance, Chapter 16.43, http://www. longbeach.gov/apps/cityclerk/lbmc/title-16/chapter-16-43.htm [accessed May 2006].

Federal Aviation Administration (FAA), 2005. FAA Stage 4 Aircraft Noise Standards, Federal Register, 70-127, pp. 38741-50.

General Accounting Office (GAO), 2000. Report to the Ranking Democratic Member, Committee on Transportation and Infrastructure, House of Representatives, Aviation and the Environment: Airport Operations and Future Growth Present Environmental Challenges, http://www.gao.gov/archive/2000/rc00153.pdf, [accessed May 2006].

Girvin, R., 2006a. Airport noise regulation, airline service quality, and social welfare: The monopoly case, Unpublished paper, Program in Transportation Science, UC Irvine.

Girvin, R., 2006b. Economic Analysis of Aircraft and Airport Noise Regulation, Unpublished Ph.D. dissertation, Program in Transportation Science, UC Irvine.

Girvin, R., 2006c. Aircraft noise abatement and mitigation strategies, Unpublished paper, Program in Transportation Science, UC Irvine.

Hsu, C.-I., LIN, P.-H., 2005. Performance assessment for airport noise charge policies and airline network adjustment response, Transportation Research Part D 10, 281-304.

Kranser, L., 2002. Chronology of the War over El Toro Airport, Internet for Activists, Writers Club Press, San Jose.

McMillen, D., 2004, Airport expansions and property values: The case of Chicago O'Hare airport, Journal of Urban Economics 55, 627-640.

Morrell, P., Lu, C., 2000. Aircraft noise social cost and charge mechanisms-A case study of Amsterdam airport Schiphol, Transportation Research Part D 5, 305-320.

Morrison, S.A., 1987. The equity and efficiency of runway pricing, Journal of Public Economics 34, 45-60. 
Morrison, S.A., Winston, C., Watson, T., 1999, Fundamental flaws of social regulation: The case of airplane noise, Journal of Law and Economics 42, 723-743.

Nelson, J.P., 2003. Meta-analysis of airport noise and hedonic property values: Problems and prospects, Journal of Transport Economics and Policy 38 (Part 1), 1-22.

Nero, G., Black, J., 2000. A critical examination of airport noise mitigation scheme and an aircraft noise charge: The case of capacity expansion and externalities at Sydney (Kingsford Smith) airport, Transportation Research Part D 5, 433-461.

Nero, G., Black, J., 1998. Hub-and-spoke networks and the inclusion of environmental costs on pricing, Transportation Research Part D 3, 275-296.

Oum, T.H., Zhang, Y., 1990. Airport pricing : Congestion tolls, lumpy investment, and cost recovery, Journal of Public Economics 43, 353-374.

Schiphol Group, 2003. Sustainable Development at Amsterdam Airport Schiphol, http:// www.schiphol.nl/media/portal/schiphol-group/pdf/pdf-files/verslag-vo-2003-uk-v1-m5657 7569830644545.pdf, [accessed May 2006].

Schipper, Y., NiJkamp, P., Rietveld, P., 1998. Why do aircraft noise value estimates differ?, Journal of Air Transport Management 4, 117-24.

Schipper, Y., Nijkamp, P., Rietveld, P., 2003. Airline deregulation and external costs: A welfare analysis, Transportation Research Part B 37, 699-718.

Wit, R.C.N., Davidson, M.D., Dings, J.M.W., 2003. Meeting external costs in the aviation industry, Report to the Commission for Integrated Transport, CE, Delft, http://www. cfit.gov.uk/docs/2003/aec/research/pdf/aec.pdf, [accessed May 2006].

Zhang, A., Zhang, Y., 2003. Airport charges and capacity expansion: effects of concessions and privatization, Journal of Urban Economics 53, 54-75. 


\section{Footnotes}

*We thank Kurt Van Dender and Ricardo Flores-Fillol for helpful comments. Any errors, however, are our responsibility.

${ }^{1}$ The U.S. General Accounting Office (2000) identified noise as the greatest environmental concern at 29 out of the 50 busiest airports.

${ }^{2}$ Nero and Black (2000) provide an analysis of the effect of per-flight taxes on an airline's operations and network structure, motivating such taxation as a noise-abatement measure. However, their model lacks the richness of the framework developed below. Schipper, Rietveld and Nijkamp (2003) also consider the noise effects of airline deregulation in a model with frequency competition, but they do not analyze noise-abatement policies. Hsu and Lin (2005) analyze noise taxation in a complex simulation-based network model.

${ }^{3}$ The FAA recently adopted a new Stage 4 aircraft noise certification standard, which represents a 10 decibel cumulative noise reduction below the current Stage 3 standard (FAA (2005)). Glasgow, Leeds, and Heathrow airports in the U.K. also set their own per-aircraft noise limits and fine operators that exceed the limits.

${ }^{4}$ For each planning year, AMS establishes an annual total noise volume, which determines the number of available slots for airline operations, based on the noise characteristics of forecast fleet composition. If actual airline operations exceed the planned noise limit, the law requires the airport to curtail operations (Schiphol Group (2003)). LGB imposes a cumulative noise exposure limit on passenger and cargo airlines. The number of permissible airline operations at LGB depends on the cumulative noise from operations in the previous year. If the prior year's noise were significantly less than the limit, the airport could increase flight slots available to the airlines. But if noise exceeded the limit, the airport would reduce the number of slots available to those airlines with the noisiest operations (City of Long Beach Ordinance, Chapter 16.43). At LHR, the noise quotas apply to nighttime operations, between 11:30 PM and $6 \mathrm{AM}$ (Wit et al. (2003)). Airlines with quieter aircraft may operate more nighttime flights than those with noisier aircraft.

${ }^{5}$ Cumulative noise is computed using a formula that can be approximated by $L=n+10 \log (f)$.

${ }^{6}$ Note that the consumer compares frequencies for the two duopoly airlines along with the fares they charge in making a choice between them, without considering the exact timing of individual flights. While this approach may not be fully accurate for individual consumers, it could apply to business travelers, who cannot predict their travel times and thus purchase refundable full-fare tickets on the airline with the best frequency/fare combination, tickets 
that allow them to board the next flight upon arriving at the airport. Alternatively, the model could capture the choice setting of a corporate travel department, which must sign an exclusive contract with a particular airline for transporting its employees. The travel department cares about the average schedule delay for the company employees, while also seeking low fares. It signs an exclusive contract with the airline providing the best combination of these features.

${ }^{7}$ While $a$ represents the gain from traveling on airline 1 relative to airline 2 , the above condition requires measurement of the absolute gain from traveling on 1. For an easy transition between the relative and absolute cases, it is assumed that when $a>0$, the absolute gain from using airline 1 equals $a$ itself while the absolute gain from airline 2 equals zero. Conversely, when $a<0$, the absolute gain from airline 1 equals 0 while the absolute gain from airline 2 equals $a$ itself.

${ }^{8}$ Using the inequality $b<p_{2}+\gamma / f_{2}$, which holds by assumption, to eliminate $b$ in (6) and using $a>\widehat{a}$, it follows that (3) is satisfied for all these consumers, indicating that airline 1 is also preferred to airline 2 .

${ }^{9}$ As seen below, the duopoly fare and frequency solutions are independent of $b$.

${ }^{10}$ Girvin's model does not rely on the present brand-loyalty framework, but instead follows the approach of Brueckner (2004), where brand loyalty is absent but the travel benefit $b$ varies across consumers. This alternate approach nevertheless yields a traffic solution with the form of (7).

${ }^{11}$ This requirement reduces to $\left(4 \gamma / \alpha^{2} f_{1}^{3}\right)\left(p_{1}-\tau\right)-(1 / \alpha)^{2}\left(\epsilon / L+\gamma / f_{1}^{2}\right)^{2}>0$.

${ }^{12}$ Since abatement cost per flight equals $\epsilon s / n$, abatement cost per seat is $\epsilon / n=\epsilon f / L$

${ }^{13}$ Recall that for the duopoly case to be relevant, the inequality $b \geq p+\gamma / f$ must hold. Substituting the $p$ and $f$ solutions from (11) and (12) yields a lower bound for $b$ in terms of the parameters of the model. An analogous lower bound arises in the other cases considered below.

${ }^{14}$ Application of the envelope theorem to the profit expression in (8) shows that airline 1's profit is increasing in $L$ holding $f_{2}$ fixed, indicating that profit falls as regulation is tightened conditional on $f_{2}$. But the equilibrium profit of each airline, found by substituting the symmetric solutions from (11) and (12) into the profit function is decreasing in $L$, indicating that tighter regulation is instead beneficial. This conclusion is counterintuitive, but it evidently arises from the competitive interaction between the airlines. 
${ }^{15}$ Although the second-order for the second-stage $p_{1}$ choice is satisfied, the second-order condition for the first-stage $f_{1}$ choice requires $f_{1}{ }^{3}<\alpha \gamma / 3 \theta^{2}$, which is assumed to hold.

${ }^{16}$ One change, however, concerns the profit impact of a tighter noise constraint. The $L$ derivative of the first-stage profit function can be shown to equal zero, so that the previous profit impact of the noise limit vanishes (see footnote 14).

${ }^{17}$ See Girvin (2006b) for details of the derivation. The second-order conditions under simultaneous choice require $f_{1}>\gamma / 2 \alpha$, or $2 \alpha^{2} / \gamma>\theta$, while the second-order condition for the first-stage choice of $f_{1}$ in the sequential case requires $f_{1}>\gamma / 3 \alpha$, or $3 \alpha^{2} / \gamma>\theta$.

${ }^{18}$ Airline profit is now independent of $\bar{n}$ under both simultaneous and sequential choice.

${ }^{19}$ As mentioned earlier, Nero and Black (2000) analyze the effect of airline taxes in a related model where noise per aircraft is fixed and taxes are levied on a per-flight basis, while Hsu and Lin (2005) analyze the effect of noise taxes in a complex simulation-based network model.

${ }^{20}$ See Girvin (2006b) for details.

${ }^{21}$ While noise taxation and cumulative noise regulation are equivalent in the model, a tax may be superior in the case where the airlines have different characteristics (cost per seat for example). In this case, the optimal cumulative noise limits would differ between the carriers, while a single noise tax would presumably be desirable (see the welfare analysis below).

${ }^{22}$ To show $\partial f / \partial \epsilon<0$, note that $(25)$ can be rewritten as

$$
\sqrt{\frac{\gamma}{8 \theta L^{2}(L / \epsilon)^{2}+4 L^{2}(L / \epsilon)}}=t
$$

Since $L$ is increasing in $\epsilon$, it follows that $L / \epsilon$ must be decreasing in $\epsilon$ to maintain this equality as $\epsilon$ rises, implying $\partial f / \partial \epsilon<0$ from (23). To show $\partial p / \partial \epsilon>0$, the term $\epsilon / n$ in the price solution can be rewritten as $2 L t$ and the previous results for $L$ used.

${ }^{23}$ For a cost-benefit analysis of the FAA noise regulation, see Morrison, Winston and Watson (1999).

${ }^{24}$ Empirical estimates of noise damage are generated through hedonic housing price models. See McMillen (2004) for a recent example and Nelson (2003) and Schipper, Nijkamp and Rietveld (1998) for up-to-date surveys. 
${ }^{25}$ It should be noted that the airport itself, which may earn profits from its operations, is not considered in the welfare analysis. Such profits would arise from unmodeled charges levied on the airlines, or from noise taxes under a tax regime. However, since airport profits represent a transfer from the airlines to the airport, they would cancel in a welfare function that equals the sum of airline and airport profits and consumer surplus. As a result, the interests of the airport can be ignored in the welfare analysis. Airport behavior, however, plays a key role in other types of models; for representative studies, see Morrison (1987), Oum and Zhang (1990), and Zhang and Zhang (2003).

${ }^{26}$ With the second-best values of both $f$ and $L$ lower than the first-best values, a comparison of the $n$ levels (given by $f / L$ ratio) is inconclusive.

${ }^{27}$ Since $W$ and none of the sequential $f$ solutions depend on $\alpha$, the value of this parameter need not be specified. Recalling that satisfaction of the airline second-order conditions in the first stage of sequential case is not guaranteed, it can then be assumed that $\alpha$ takes a value such that these conditions hold.

${ }^{28}$ The full set of numerical results is available in Girvin (2006b). In contrast to Proposition 7, numerical examples in Girvin (2006a) show that, in the monopoly case, cumulative noise regulation is slightly superior to per-aircraft regulation everywhere in her chosen parameter space. This finding, of course, does not establish a general result.

${ }^{29}$ These aircraft include the Boeing 777, 737-700 to -900, and 717, along with the Airbus A320/321, A330 and A340. 